\title{
T Cells, Endothelial Cell, Metabolism; A Therapeutic Target in Chronic Inflammation
}

\section{Li ZP, Yang J, Pau CW, Wong PC, Deng C and Cheung CPK*}

The School of Life Sciences, The Chinese University of Hong Kong, Hong Kong

*Corresponding author: Kenneth CP Cheung, The School of Life Sciences, The Chinese University of Hong Kong, Hong Kong, Email: c.cheung@cuhk.edu.hk

\section{Review Article}

Volume 5 Issue 2

Received Date: June 19, 2020

Published Date: July 03, 2020

DOI: $10.23880 /$ oajmb-16000163

\section{Abstract}

The role of metabolic reprogramming in the coordination of the immune response has gained increasing consideration in recent years. Indeed, it has become clear that changes in the metabolic status of immune cells can alter their functional properties. During inflammation, stimulated immune cells need to generate sufficient energy and biomolecules to support growth, proliferation and effector functions, including migration, cytotoxicity and production of cytokines. Thus, immune cells switch from oxidative phosphorylation to aerobic glycolysis, increasing their glucose uptake. A similar metabolic reprogramming has been described in endothelial cells which have the ability to interact with and modulate the function of immune cells and vice versa. Nonetheless, this complicated interplay between local environment, endothelial and immune cells metabolism, and immune functions remains incompletely understood. We analyze the metabolic reprogramming of endothelial and T cells during inflammation and we highlight some key components of this metabolic switch that can lead to the development of new therapeutics in chronic inflammatory disease.

Keywords: Endothelial Cells; Peptide Antigens; Ragulator Complex; Glutaminolysis

Abbreviations: Ecs: Endothelial Cells; Cams: CellAdhesion Molecules; TNF: Tumor Necrosis Factor; Tcrs: T Cell Receptors; MHC: Major Histocompatibility Complex; Apcs: Antigen-Presenting Cells; Dcs: Dendritic Cells; NF: Nuclear Factor; VEGF: Vascular Endothelial Growth Factor; TCA: Tricarboxylic Acid; OXPHOS: Oxidative Phosphorylation; PPP: Pentose Phosphate Pathway.

\section{Introduction}

Endothelial cells (ECs) make up the luminal surface of blood vessels and play an active role in recruiting, activating and regulating leukocytes by expressing cell-adhesion molecules (CAMs) and cytokines [1]. Interactions between endothelial cells and T cells are bi-directional. Endothelial cells display class I and class II MHC-peptide complexes on their surface and come in regular contact with circulating $\mathrm{T}$ cells. In return, activated $\mathrm{T}$ cells differentiate into three different known effectors - $\mathrm{T}$ helper cells (CD4+ $\mathrm{T}$ cells), cytotoxic T cells (CD8+ cells) and T regulatory (Treg) cells, all of which have different functionalities in mediating immune responses, such as providing both soluble and contactdependent signals to modulate and protect endothelial cells.

Studies showed that activation of ECs and differentiation of activated $\mathrm{T}$ cells requires distinct metabolic pathways. The different factors that regulate metabolism in cells are highly intertwined, and there has been increased interest in how EC and $\mathrm{T}$ cell metabolism are reprogrammed during inflammation.

In this review, we provide an overview of the basic metabolic reprogramming of endothelial and $T$ cells in inflammation upon activation, the metabolic reprogramming that will be mentioned includes glycolysis, the pentose phosphate pathway, the TCA cycle, as well as both fatty acid and amino acid metabolism. After establishing this 


\section{Open Access Journal of Microbiology \& Biotechnology}

background, we discuss the potential targets in each metabolic pathway for therapies in inflammation.

\section{The T Cell-Endothelium Immunological Synapse}

\section{Endothelial Cells Modulation by T Cells}

Inflammation is a tissue response initiated by host defense mechanisms. While inflammation is the bodily response to get rid of invading pathogens and promotes the recovery of damaged tissues, uncontrolled chronic inflammation can be the center of many diseases. In inflammation, ECs play a key role in mediating immune cells trafficking. ECs recruit immune cells to the site of injury via vasodilation. In return, immune cells accumulate at the injure site by rolling along and adhering firmly on the endothelium before migrating across the blood vessel. This is a process known as diapedesis [2]. Inflammatory cytokines interleukin (IL)-1 $\beta$ and tumor necrosis factor (TNF)- $\alpha$ are responsible for regulating endothelial cell responses to inflammation [3]. The development of tissue remodeling is also attributed by the two cytokines [4]. Nuclear factor (NF)- $\mathrm{BB}$, which is a transcription factor, mediates downstream signaling of IL-1 $\beta$ and TNF- $\alpha$. It also contributes to inflammation, cell proliferation, differentiation, apoptosis, as well as cell survival $[3,5]$. NF- $\mathrm{kB}$ has involvement in numerous important functions of endothelium such as angiogenesis. The role of $\mathrm{NF}-\kappa \mathrm{B}$ in the mediation of endothelial cells in tissue repair has however not been reported.

\section{Regulation of $T$ Cells Activation and Differentiation by Endothelial Cells}

$\mathrm{T}$ cells are a part of the adaptive immune system that is responsible for surveying tissues for the presence of infecting pathogens, it is also responsible for killing these cells when triggered. Through $\mathrm{T}$ cell receptors (TCRs), they can detect non-self-protein fragments called peptide antigens (Ag). These peptide antigens can only be sensed when they are presented on the surface of antigen-presenting cells (APCs, such as dendritic cells, macrophages and B cells) by major histocompatibility complex (MHC) molecules. When naive T cells are activated by dendritic cells (DCs), they are directed by pivotal cytokines to differentiate into different types of $\mathrm{T}$ cells: CD4 T helper (Th cells), CD8 T cells (cytotoxic T cells) and Treg cells. CD4 T cells only response to Ag presented on class II MHC molecules (MHC-II). These cells aid in immune responses by secreting cytokines that "help" other immune cells to conduct their specific functions. CD8 T cells respond to Ag presented on MHC-I and participate in the direct killing of Ag-presenting cells.

The process of $\mathrm{T}$ cell activation and differentiation requires a broad spectrum of trafficking patterns and interactions with endothelial cells. It has been proposed that ECs can induce Treg proliferation and enhance Treg suppressive capacities but there is limiting evidence in both humans and chronic inflammatory models. It is later hypothesized that endothelial cells could potentially modulate $\mathrm{T}$ cell function in relation to skin chronic diseases under chronic inflammatory cytokine activation.

\section{Metabolic Pathways in Endothelial and T Cells Activation}

\section{Glycolysis}

Based on their morphology and role in angiogenesis, ECs can be classified into three different subtypes: the highly branched tip cells, which primarily migrate and navigate the direction of the vessel sprout, the stalk cells, which have less branches but are highly proliferative and elongate the sprout during extension, and the phalanx cells, which are commonly recognized by their cobblestone appearance, line the mature blood vessels and are passive [2]. Due to its low mitochondrial content, ECs rely mostly on glycolysis for energy production. Upon vascular endothelial growth factor (VEGF) stimulation, the glycolytic rate is doubled and the expression of GLUT1 is up-regulated in order to meet the metabolic demands of cell migration to the site of injury [2]. PFKFB3, an isoform of 6-phosphofructo-2-kinase capable of metabolizing the glycolytic activator fructose-2,6-biphosphate, is also involved in the metabolic regulation of ECs. Glycolysis is shown to modulate the phenotypic expression of ECs. Predestined stalk cells could be genetically override into metabolically active tip cells to further enhance ECs sprouting, and this can be done by up-regulating the activity of PFKFB3 [6]. To conclude, glycolysis is critical for ECs, and its complete absence results in decreased proliferation and migration, as long as the induction of cell death.

In the field of immunometabolism, recent findings show that the metabolic status of immune cells can determine the type of immune response induced. The traditional concept of energy production is that, through the process of glycolysis, two molecules of ATPs are generated from one molecule of glucose. The metabolism of glycolysis first converts glucose to pyruvate, which then enters the mitochondrial tricarboxylic acid (TCA) cycle to generate carbon dioxide, NADH and FADH2. The reducing equivalents NADH and FADH2 drive oxidative phosphorylation (OXPHOS) for more ATP synthesis.

It is known that a switch from OXPHOS to aerobic glycolysis is a hallmark for T cell activation and is thought to be the metabolic requirement for proliferation [7]. In the 1920 s, it was shown that, even in aerobic conditions, 


\section{Open Access Journal of Microbiology \& Biotechnology}

cancer cells can metabolize about ten times more glucose to produce lactate than normal tissues can. This is known as the Warburg effect [8]. In recent findings, it shows that the Warburg effect-like metabolic reprogramming also exists in immune cells, especially in $\mathrm{T}$ cells, and determines the function of the immune cell subsets in disease conditions such as those in inflamed tissue. Specifically, it was shown that OXPHOS and aerobic glycolysis interchangeably fuel $\mathrm{T}$ cell proliferation and survival, but only aerobic glycolysis can facilitate full effector status [7].

The T effector subsets, Th1, Th2 and Th17, are known to prefer glycolysis even in aerobic conditions [2]. Upon encountering antigens, $\mathrm{T}$ cells increase rates of glucose uptake dramatically by inducing Glut 1 trafficking to the cell surface; phosphorylation of glycolytic enzyme activity, and the activation of mammalian target of rapamycin (mTOR). Tuberous sclerosis complex-2, which is an inhibitor of mTOR is phosphorylated by Akt and this triggers its proteasomal degradation and inhibits the repressing mTOR activity. mTOR is significant to the increase in glycolysis in activated T cells, and is therefore a key regulator of cellular metabolism [9].

\section{The Pentose Phosphate Pathway}

Apart from being metabolized in the glycolytic pathway to generate ATP, glucose can also enter the pentose phosphate pathway (PPP), which is used to fuel the synthesis of ribose5-phosphate, which is essential for the biosynthesis of nucleotides. The PPP consists of both oxidative (oxPPP) and non-oxidative branch (non-oxPPP) [10], and studies showed that the inhibition of either of these branches impairs the viability and migration of ECs [11]. Glucose-6-phosphate dehydrogenase, whose activity is controlled by VEGF, controls the flux going through the oxPPP. Glycolytic intermediates can also enter the serine biosynthesis pathway, which is required for proliferation and survival in ECs due to its importance in both heme and nucleotide synthesis [12].

\section{The TCA Cycle}

The TCA cycle is one crucial part that generates many metabolic intermediates for the de novo synthesis of nucleotides, proteins and lipids in many proliferating cell types [13]. Upon $\beta$-oxidation in the mitochondria, long chain fatty acids (FAs) produce acetyl-CoA. Carnitine palmitoyl transferase 1 alpha (CPT1A), which is the rate-limiting enzyme of FAO, is responsible for controlling the transport of FAs into the mitochondria [14]. In ECs, many TCA cycle intermediates have FA-derived carbons, while loss of CTP1A resulted in defects in endothelial sprouting, which was due to decreased biomass synthesis, in particular, reduced deoxyribonucleotide (dNTP) synthesis. In the same study, acetyl-CoA derived from FA was found to be the major source of carbon for TCA cycle intermediates including citrate, $\alpha$-ketogluterate $(\alpha \mathrm{KG})$, glutamate, and aspartate, which is a crucial carbon source for dNTP synthesis [15]. In the same study, it was shown that CPT1A knockdown in cultured ECs severely dampened the contribution of FA-served carbon to dNTPs and lowered total levels of dNTPs produced [15]. In return, there was decreased proliferation of ECs in vitro and reduced EC proliferation in the developing retinal vascular network, which led to decreased sprout length and branching complexity in vivo [15]. In conclusion, FA oxidation exclusively contributes to non-lipid biomass in ECs as recent evidence shows that FAO does not provide carbon to nonlipid biomass in many other cell types [16].

Apart from the mentioned changes in glycolysis, mitochondrial function is also enhanced in activated T cells. Not only do activated $T$ cells need the oxidation of glucosederived acetyl-CoA and the integration of glucose and fatty acid metabolites, both by the TCA cycle, but they also consume glutamine to support the production of biomass and ATP [2]. Upon activation, the availability of glutamine is crucial for T-cell survival, proliferation and effector functioning [17]. Activated $\mathrm{T}$ cells increases expression of glutamine transporters to uptake more glutamine, there is also increase in production of enzymes involved in glutaminolysis [17]. CD28-ERK signaling mediates the above changes mentioned, while the induction of $M y c$ expression is required [18]. Here, glutamine is required for full mTOR activation [15], which is possibly related to its role in the facilitation of direct mTOR complex I (mTORC1) activation by other amino acids $[19,20]$. Furthermore, when glucose is scarce, glutamine fuels the TCA cycle and help maintain the abundance of key components such as pyruvate and citrate [21].

In addition, acetate can enter the TCA cycle after the conversion to acetyl-CoA. It is observed that this pathway has important implications for T-cell effector functions. Upon infection, increase in systemic acetate levels was noted. Once acetate is uptake into CD8 memory T cells, it enters the TCA cycle and expands the citrate-derived acetyl-CoA pool, which promotes the post-translational acetylation of GAPDH. In return, efficiency is increased and IFN- $\gamma$ production is interlinked.

\section{Fatty Acid Metabolism}

Adenosine monophosphate-activated protein kinase (AMPK)activityinECssenseglucose deprivation and promotes the inhibition of acetyl-coenzyme a carboxylate (ACC), which results in increased FAO [22]. Studies investigated FAO in ECs and found it crucial for EC proliferation during vessel sprouting, as opposed to glycolysis that regulates both proliferation and migration [15]. 


\section{Open Access Journal of Microbiology \& Biotechnology}

Likewise, T-cell clonal expansion requires significant synthesis of cellular and organelle membranes, this means the availability of lipid metabolites, including cholesterol, is especially important. In addition, upon encountering antigen-presenting cells (APCs), naïve T cells are activated to undergo clonal expansion and differentiation, and rely on fatty acid oxidation (FAO) and OXPHOS for their energy production. At the single cell level, regulation of cholesterol metabolism has important implications for TCR clustering and signaling. Moreover, a subset of $\mathrm{T}$ cells, Treg cells, mainly prefers FAO for energy generation and replicates at moderate levels unlike the profound amplification in $\mathrm{T}$ effector cell proliferation [2]. Inhibitors of 3-hydroxyl-3methyl-glutaryl-coenzyme, which is the limiting enzyme of the cholesterol biosynthesis pathway, can counteract the mTORC1 suppressive function. This was in association with reduced Treg cell proliferation, impaired expression of surface CTLA-4 and inducible T-cell co-stimulator, which regulate cell-contact dependent suppressive mechanisms.

\section{Amino Acid Metabolism}

Apart from up-regulation of aerobic glycolysis and PPP, which is attained via glucose transporter (GLUT) 1 expression at the cell periphery and up-regulation of glycolytic enzymes, glutamine metabolism is also enhanced and this supplies products for the TCA cycle and promote polyamide synthesis. Once glutamine enters the cell, glutaminase (GLS) metabolizes it to glutamate. Glutamate is then converted to $\alpha \mathrm{KG}$ by glutamate dehydrogenase (GDH) and plays a part in the synthesis of protein and nucleotides by entering the TCA cycle.

Besides glutamine, activated $\mathrm{T}$ cells also increase their intake of large neutral amino acids. In a study to demonstrate the importance of this uptake, the system $\mathrm{L}$ transporter S1c7a5 (LAT1), which is responsible for transporting arginine, leucine, phenylalanine, tyrosine and tryptophan, was genetically knocked-out in murine $\mathrm{T}$ cells [23]. It was shown that LAT1-deficient CD4 $\mathrm{T}$ cells became unable to proliferate and to differentiate in vitro into Th1 or Th17 cells. Moreover, CD8 T-cell proliferation and differentiation were significantly impaired both in vitro and in vivo. However, there was no effect to the differentiation to Tregs. Large neutral amino acids, specifically arginine and leucine, are important for the signaling of amino acid sufficiency to mTOR complex 1 (mTORC1), which allows its activation. The pathway involves the RAS-related GTP binding protein (Rag) family of small GTPases. With amino acids present, Rag translocates mTORC1 A/B-dependently to the lysosomal membrane, which brings it close to its activator Rheb [24]. The Rags, which are located to the lysosome by interacting with the pentameric 'Ragulator complex', consists of the 'late endosomal/lysosomal adaptor and MAPK and mTOR activator' proteins 1-5 (LAMTOR 1-5) [25]. The protein GATOR2 seems to be the critical hub in the upstream molecular regulation of Rag A/B activation by specific amino acid. It positively regulates Rag A/B activity. It self however is inhibited when interacting with either Sestrin2 or CASTOR 1, where the presence of arginine or leucine competitively disrupts these interactions respectively [2628]. During T-cell activation, there is up-regulation of both uptake of amino acids and elements used in this molecular pathway, which dramatically promotes mTORC1 activity when acting together. Upon activation of $\mathrm{T}$ cells, both the recruitment of mTORC1 to the lysosome and its activity are enhanced. They are equally important to the differentiation of CD4 T cells into Th1 effector cells, which can be dampened by pharmacological mTORC1 inhibition [29]. AA-sensing capacity has been linked to T-cell fate. Cells with more of the transporter showed a higher level of recruitment and activity of mTORC lysosome, and higher levels of c-Myc expression. This results in increased glycolytic and mitochondrial respiratory capacity, better proliferation and effector cytokine production [30].

\section{Conclusion}

To cope with the increased bioenergetic and biosynthetic demand of proliferation, both endothelial cells and $\mathrm{T}$ cells rewire their metabolism associated with migration and proliferation. Specifically, T cells reprogram their metabolic pathways from fatty acid b-oxidation and pyruvate oxidation via the TCA cycle to the glycolytic, pentose-phosphate, and glutaminolytic pathways. Considering the importance of endothelial cells and immune cell functions in the promotion and suppression of various types of disease progressions, the ability to manipulate both endothelial and immune cells from the potential metabolic targets mentioned above shows a potential of becoming novel therapeutic approaches.

\section{References}

1. Lim W, Olding M, Healy E, Millar TM (2018) Human Endothelial Cells Modulate CD4+ T Cell Populations and Enhance Regulatory T Cell Suppressive Capacity. Front Immunol 9: 565.

2. Tang C, Mauro C (2017) Similarities in the Metabolic Reprogramming of Immune System and Endothelium. Front Immunol 8: 837.

3. Saklatvala J, Davis W, Guesdon F (1996) Interleukin 1 (IL1) and Tumour Necrosis Factor (TNF) Signal Transduction. Philos Trans R Soc Lond B Biol Sci 351(1336): 151-157.

4. Chaudhuri V, Zhou L, Karasek M (2007) Inflammatory cytokines induce the transformation of human dermal 


\section{Open Access Journal of Microbiology \& Biotechnology}

microvascular endothelial cells into myofibroblasts: a potential role in skin fibrogenesis. J Cutan Pathol 34(2): 146-153.

5. Wu JT, Kral JG (2005) The NF-kappaB/IkappaB signaling system: a molecular target in breast cancer therapy. J Surg Res 123(1): 158-169.

6. De Bock Katrien, Georgiadou M, Gerhardt H, Dewerchin M, Carmeliet P, et al. (2013) Role of PFKFB3-Driven Glycolysis in Vessel Sprouting. Cell 154(3): 651-663.

7. Chang CH, Curtis JD, Pearce EL, Jones RG, Maggi LB, et al. (2013) Posttranscriptional control of T cell effector function by aerobic glycolysis. Cell 153(6): 1239-1251.

8. Warburg O, Wind F, Negelein E (1927) The metabolism of tumors in the body. J Gen Physiol 8(6): 519-530.

9. Frauwirth KA, Thompson CB (2004) Regulation of T Lymphocyte Metabolism. J Immunol 172(8): 4661-4665.

10. Pandolfi PP, Sonati F, Rivi R, Mason P, Grosveld F, et al. (1995) Targeted disruption of the housekeeping gene encoding glucose 6-phosphate dehydrogenase (G6PD): G6PD is dispensable for pentose synthesis but essential for defense against oxidative stress. The EMBO Journal 14(21): 5209-5215.

11. Vizan P, Tena SS, Vizan GA, Soler M, Messeguer R, et al. (2009) Characterization of the metabolic changes underlying growth factor angiogenic activation: Identification of new potential therapeutic targets. Carcinogenesis 30(6): 946-952.

12. Vandekeere S, Dubois C, Kalucka J, Sullivan MR, Carmeliet P, et al. (2018) Serine Synthesis via PHGDH is Essential for Heme Production in Endothelial Cells. Cell Metab 28(4): 573-587.

13. Natalya PN, Thompson CB (2016) The Emerging Hallmarks of Cancer Metabolism. Cell Metab 23(1): 2747.

14. Eaton S (2002) Control of Mitochondrial beta-oxidation flux. Prog Lipid Res 41(3): 197-239.

15. Schoors S, Bruning U, Missiaen R, Queiroz KCs, Borgers G, et al. (2015) Fatty Acid Carbon is Essential for dNTP Synthesis in Endothelial Cells. Nature 520(7546): 192197.

16. Hosios AM, Hecht VC, Danai LV, Steinhauser ML, Manalis SR, et al. (2016) Amino Acids Rather than Glucose Account for the Majority of Cell Mass in Proliferating Mammalian Cells. Dev Cell 36(5): 540-549.
17. Nakaya M, Xiao $Y$, Zhou X, Chang JH, Chang M, et al. (2014) Inflammatory $T$ cell responses rely on amino acid transporter ASCT2 facilitation of glutamine uptake and mTORC1 kinase activation. Immunity 40(5): 692-705.

18. Wang R, Dillon CP, Shi LZ, Milasta S, Carter R, et al. (2011) The transcription factor Myc controls metabolic reprogramming upon Tlymphocyte activation. Immunity 35(6): 871-872.

19. Nicklin P, Bergman P, Zhang B, Triantafellow E, Wang $\mathrm{H}$, et al. (2009) Bidirectional transport of amino acids regulates mTOR and autophagy. Cell 136(3): 521-534.

20. Duran RV, Oppliger W, Robitaille AM, Heiserich L, Skendaj $\mathrm{R}$, et al. (2012) Glutaminolysis activates Rag-mTORC1 signaling. Mol Cell 47(3): 349-358.

21. Blagih J, Coulombe F, Vincent EE, Dupuy F, Galicia-Vazquez G, et al. (2015) The energy sensor AMPK regulates T cell metabolic adaptation and effector responses in vivo. Immunity 42(1): 41-54.

22. Zeina D, Ruderman N, Keith T, Yasuo I (2001) Circulation Research 88: 1276-1282.

23. Zeng H, Yang K, Cloer C, Neale G, Vogel P, et al. (2013) mTORC1 couples immune signals and metabolic programming to establish Treg-cell function. Nature 499(7459): 485-490.

24. Long X, Ortiz-Vega S, Lin Y, Avruch J (2005) Rheb binding to mammalian target of rapamycin (mTOR) is regulated by amino acid sufficiency. J Biol Chem 280(25): 2343323436.

25. Sancak Y, Bar-Peled L, Zoncu R, Markhard AL, Nada S, et al. (2010) Ragulator-Rag complex targets mTORC1 to the lysosomal surface and is necessary for its activation by amino acids. Cell 141(2): 290-303.

26. Saxton RA, Knockenhauer KE, Wolfson RL, Chantranupong L, Pacold ME, et al. (2016) Structural basis for leucine sensing by the Sestrin2-mTORC1 pathway. Science 351(6268): 53-58.

27. Wolfson RL, Chantranupong L, Saxton RA, Shen K, Scaria $\mathrm{SM}$, et al. (2016) Sestrin2 is a leucine sensor for the mTORC1 pathway. Science 351(6268): 43-48.

28. Chantranupong L, Scaria SM, Saxton RA, Gygi MP, Shen K, et al. (2016) The CASTOR proteins are arginine sensors for the mTORC1 pathway. Cell 165(1): 153-164.

29. Kolev M, Dimeloe S, Le Friec G, Navarini A, Arbore G, et al. (2015) Complement regulates nutrient influx and metabolic reprogramming during Th1 cell responses. 


\section{Open Access Journal of Microbiology \& Biotechnology}

Immunity 42(6): 1033-1047.

30. Pollizzi KN, Sun IH, Patel CH, Lo YC, Waickman AT, et al. (2016) Asymmetric inheritance of mTORC1 kinase activity during division dictates CD8+ $\mathrm{T}$ cell differentiation. Nat Immunol 17(6): 704-11.

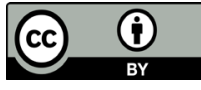

